\title{
The masses and axial currents of the doubly charmed baryons
}

\author{
Zhi-Feng Sun ${ }^{1,2, *}$ Zhan-Wei Liu ${ }^{3}{ }^{\dagger}$ Xiang Liu ${ }^{1,2},{ }^{\dagger}$ and Shi-Lin Zhu ${ }^{4,5,6 \S}$ \\ ${ }^{1}$ School of Physical Science and Technology, Lanzhou University, Lanzhou 730000, China \\ ${ }^{2}$ Research Center for Hadron and CSR Physics, Lanzhou University and Institute of Modern Physics of CAS, Lanzhou 730000, China \\ ${ }^{3}$ CSSM, School of Chemistry and Physics, University of Adelaide, Adelaide, South Australia 5005, \\ Australia ${ }^{4}$ School of Physics and State Key Laboratory of Nuclear Physics and Technology, Peking University, Beijing 100871, China \\ ${ }^{5}$ Collaborative Innovation Center of Quantum Matter, Beijing 100871, China \\ ${ }^{6}$ Center of High Energy Physics, Peking University, Beijing 100871, China
}

\begin{abstract}
The chiral dynamics of the doubly heavy baryons is solely governed by the light quark. In this work, we have derived the chiral corrections to the mass of the doubly heavy baryons up to $\mathrm{N}^{3} \mathrm{LO}$. The mass splitting of $\Xi_{c c}$ and $\Omega_{c c}$ at the $\mathrm{N}^{2} \mathrm{LO}$ depends on one unknown low energy constant $c_{7}$. By fitting the lattice masses of $\Xi_{c c}(3520)$, we estimate the mass of $\Omega_{c c}$ to be around $3.726 \mathrm{GeV}$. Moreover, we have also performed a systematical analysis of the chiral corrections to the axial currents and axial charges of the doubly heavy baryons. The chiral structure and analytical expressions will be very useful to the chiral extrapolations of the future lattice QCD simulations of the doubly heavy baryons.
\end{abstract}

PACS numbers: 14.40.Rt, 14.40.Lb, 12.39.Hg, 12.39.Pn

\section{INTRODUCTION}

As one of the most important groups in the baryon family, the doubly charmed baryons are composed of two charmed quarks and one light quark (the doubly heavy baryons $\Xi_{c c}^{++}$, $\Xi_{c c}^{+}$and $\Omega_{c c}^{+}$with quark components $c c u, c c d$ and $c c s$, respectively), which were predicted in the quark model (see Ref. [1] for a detailed review). In the past decades, there have been some experimental efforts in the search of the doubly charmed baryons [2-5]. The SELEX Collaboration announced the first observation of the doubly charmed baryon $\Xi_{c c}^{+}(3520)$ with the mass $M=3519 \pm 1 \mathrm{MeV}$ and width $\Gamma=3 \mathrm{MeV}$ [2], where the observed decay mode is $\Xi_{c c}^{+} \rightarrow \Lambda_{c}^{+} K^{-} \pi^{+}$. Later, $\Xi_{c c}^{+}(3520)$ was confirmed by SELEX in the $p D^{+} K^{-}$decay channel with the mass $3518.7 \pm 1.7 \mathrm{MeV}$ [4]. Although SELEX also reported $\Xi_{c c}^{+}(3520)$, these results were not confirmed by FOCUS [6], BaBar [7], Belle [8] and LHCb collaborations [9].

The doubly charmed baryons have been extensively studied with different theoretical approaches. The $\Xi_{c c}$ mass was predicted to be $3.48 \sim 3.74 \mathrm{GeV}$ in the quark model, while the $\Omega_{c c}$ mass is estimated to be $3.59 \sim 3.86 \mathrm{GeV}$ [10-22]. The Lattice QCD groups also studied these systems [23-27], where the predicted mass of $\Xi_{c c}$ is $3.51 \sim 3.67 \mathrm{GeV}$ and the mass of $\Omega_{c c}$ is $3.68 \sim 3.76 \mathrm{GeV}$.

The mass splittings of baryons within the same multiplet encode important information on their inner structure. For example, the mass splittings of the light baryons were reviewed in Refs. [28, 29]. In Refs. [30, 31], the mass splitting of the singly heavy baryons was studied within the framework of the chiral perturbation theory. In Ref. [32], the authors investigated the mass splitting of the doubly heavy baryons by considering the heavy diquark symmetry. Besides the baryon

\footnotetext{
*Electronic address: sunzhif09@1zu.edu.cn

†Electronic address: zhan-wei.liu@adelaide.edu.au

‡Electronic address: xiangliu@1zu.edu.cn

§Electronic address: zhusl@pku.edu.cn
}

mass, the axial current and axial charge of the baryons are also very important observables, which attract lots of attention [33-55].

The experimental search of the doubly charmed baryons is full of challenges and opportunities. In this work, we adopt the chiral perturbation theory to calculate the chiral corrections to the doubly charmed baryon masses and their mass splittings, which will be helpful to further experimental exploration of the doubly charmed baryons. Under the same framework, we also study the chiral corrections to the axial charge and axial current of the doubly charmed baryons, which may be measured through the semileptonic decays of the doubly charmed baryons in the future.

Chiral perturbation theory $(\chi \mathrm{PT})$ is an elegant framework to deal with the low energy process in hadron physics. With the help of the chiral power counting scheme proposed by Weinberg et al. [56,57], one can consider the chiral corrections to the physical observables order by order.

In the baryon sector, the baryon mass does not vanish in the chiral limit. This inherent mass scale breaks the naive chiral power counting. To solve this issue, various schemes were proposed such as the heavy baryon $\chi \mathrm{PT}$, infrared baryon $\chi \mathrm{PT}$, and extended on-mass-shell method etc.

In the heavy baryon $\chi \mathrm{PT}$, the baryon is treated to be extremely heavy and acts as a static source [54], which allows us to take the non-relativistic limit of the fully relativistic theory and make expansion in powers of the inverse baryon mass. For the case of infrared regularization, the loop integral can be separated into infrared regular part and infrared singular one $[58,59]$, where the later one conserves the Weinberg's power counting rule. In the extended on-mass-shell method, the power counting breaking terms are subtracted and the low energy constants are redefined [60-63]. In our work, we use the heavy baryon $\chi \mathrm{PT}$ approach to investigate the chiral corrections to the masses and axial currents of the doubly charmed baryons.

This paper is organized as follows. After the introduction, we introduce the chiral Lagrangians of the doubly charmed baryons and its non-relativistic reduction in Sec. II. Then we 
present the calculation details of the chiral corrections to the masses and axial currents of the doubly charmed baryons and the corresponding numerical results in Secs. III and IV respectively. This work ends with a summary in Sec. V. We collect the $\mathrm{N}^{3} \mathrm{LO}$ chiral corrections to the masses and some lengthy expressions in the appendix.

\section{THE CHIRAL LAGRANGIANS OF THE DOUBLY CHARMED BARYONS}

In order to calculate the chiral corrections to the masses and axial currents, we need construct the chiral effective Lagrangians of the doubly charmed baryons with the help of chiral, parity and charge conjugation symmetries. We firstly introduce the notations $U$ and $u$ to describe the psudoscalar meson field, which have the relation

$$
U=u^{2}=\exp \left(i \frac{\phi(x)}{F_{0}}\right),
$$

where $\phi(x)$ has the definition

$$
\phi(x)=\left(\begin{array}{ccc}
\pi^{0}+\frac{1}{\sqrt{3}} \eta & \sqrt{2} \pi^{+} & \sqrt{2} K^{+} \\
\sqrt{2} \pi^{-} & -\pi^{0}+\frac{1}{\sqrt{3}} \eta & \sqrt{2} K^{0} \\
\sqrt{2} K^{-} & \sqrt{2} \bar{K}^{0} & -\frac{2}{\sqrt{3}} \eta
\end{array}\right) .
$$

The doubly heavy baryon field $\psi$ with spin $\frac{1}{2}$ is a column vector in the flavor space, i.e.

$$
\psi=\left(\begin{array}{c}
\Xi_{c c}^{++} \\
\Xi_{c c}^{+} \\
\Omega_{c c}^{+}
\end{array}\right)
$$

where the quark contents of $\Xi_{c c}^{++}, \Xi_{c c}^{+}$, and $\Omega_{c c}^{+}$are $c c u, c c d$, and ccs, respectively.

TABLE I: The properties of the building blocks under the $S U(3)_{L} \times$ $S U(3)_{R}(\mathrm{CH})$, parity $(\mathrm{P})$ and charge conjugation (C) transformations.

\begin{tabular}{ccccccc}
\hline & $U$ & $u$ & $\chi$ & $f_{\mu \nu}^{R}$ & $f_{\mu \nu}^{L}$ & $D_{\mu} \psi$ \\
\hline $\mathrm{CH}$ & $V_{R} U V_{L}^{\dagger}$ & $V_{R} u K^{\dagger}$ & $V_{R} \chi V_{L}^{\dagger}$ & $V_{R} f_{\mu \nu}^{R} V_{R}^{\dagger}$ & $V_{L} f_{\mu \nu}^{L} V_{L}^{\dagger}$ & $K D_{\mu} \psi$ \\
$\mathrm{P}$ & $U^{\dagger}$ & $u^{\dagger}$ & $\chi^{\dagger}$ & $f^{L \mu \nu}$ & $f^{R \mu \nu}$ & $\gamma^{0} D^{\mu} \psi$ \\
$\mathrm{C}$ & $U^{T}$ & $u^{T}$ & $\chi^{T}$ & $-\left(f_{\mu \nu}^{L}\right)^{T}$ & $-\left(f_{\mu \nu}^{R}\right)^{T}$ & $C D_{\mu}^{\prime T} \bar{\psi}^{T}$ \\
\hline & $\psi$ & $\bar{\psi}$ & $\chi_{ \pm}$ & $f_{\mu \nu}^{ \pm}$ & $u_{\mu}$ & $\Gamma_{\mu}$ \\
\hline $\mathrm{CH}$ & $K \psi$ & $\bar{\psi} K^{\dagger}$ & $K \chi_{ \pm} K^{\dagger}$ & $K f_{\mu \nu}^{ \pm} K^{\dagger}$ & $K u_{\mu} K^{\dagger}$ & $K \Gamma^{\mu} K^{\dagger}-\partial^{\mu} K K^{\dagger}$ \\
$\mathrm{P}$ & $\gamma^{0} \psi$ & $\bar{\psi} \gamma^{0}$ & $\pm \chi_{ \pm}$ & $\pm f^{ \pm \mu \nu}$ & $-u^{\mu}$ & $\Gamma^{\mu}$ \\
$\mathrm{C}$ & $C \bar{\psi}^{T}$ & $\psi^{T} C$ & $\chi_{ \pm}^{T}$ & $\mp\left(f_{\mu \nu}^{ \pm}\right)$ & $\left(u_{\mu}\right)^{T}$ & $-\left(\Gamma_{\mu}\right)^{T}$ \\
\hline
\end{tabular}

In Table I, we show the transformation properties of the building blocks, which include $\chi, \chi_{ \pm}, f_{\mu \nu}^{R}, f_{\mu \nu}^{L}, f_{\mu \nu}^{ \pm}, u_{\mu}, \Gamma_{\mu}, D_{\mu}$ and $D_{\mu}^{\prime}$ with the definitions

$$
\begin{aligned}
\chi & =2 B_{0}(s+i p) \\
\chi_{ \pm} & =u^{\dagger} \chi u^{\dagger} \pm u \chi^{\dagger} u \\
f_{\mu \nu}^{R} & =\partial_{\mu} r_{v}-\partial_{v} r_{\mu}-i\left[r_{\mu}, r_{v}\right]
\end{aligned}
$$

$$
\begin{aligned}
f_{\mu \nu}^{L} & =\partial_{\mu} l_{v}-\partial_{v} l_{\mu}-i\left[l_{\mu}, l_{v}\right], \\
f_{\mu \nu}^{ \pm} & =u^{\dagger} f_{\mu \nu}^{R} u \pm u f_{\mu v}^{L} u^{\dagger}, \\
u_{\mu} & =i\left[u^{\dagger}\left(\partial_{\mu}-i r_{\mu}\right) u-u\left(\partial_{\mu}-i l_{u}\right) u^{\dagger}\right], \\
\Gamma_{\mu} & =\frac{1}{2}\left[u^{\dagger}\left(\partial_{\mu}-i r_{\mu}\right) u+u\left(\partial_{\mu}-i l_{u}\right) u^{\dagger}\right], \\
D_{\mu} & =\partial_{\mu}+\Gamma_{\mu}-i v_{\mu}^{(s)}, \\
D_{\mu}^{\prime} & =\partial_{\mu}-\Gamma_{\mu}+i v_{\mu}^{(s)},
\end{aligned}
$$

where $r_{\mu}=v_{\mu}+a_{\mu}, l_{\mu}=v_{\mu}-a_{\mu}$, and $v_{\mu}, v_{\mu}^{(s)}, a_{\mu}, s, p$ are external $c$-number fields. Considering the transformation properties listed in Table I, the chiral Lagrangian of the doubly heavy baryon can be constructed order by order, i.e.,

$$
\begin{aligned}
\mathcal{L}^{(1)}= & \bar{\psi}\left(i \not D-m+\frac{g_{A}}{2} \gamma^{\mu} \gamma_{5} u_{\mu}\right) \psi \\
\mathcal{L}^{(2)}= & c_{1} \bar{\psi}\left\langle\chi_{ \pm}\right\rangle \psi-\left\{\frac{c_{2}}{8 m^{2}} \bar{\psi}\left\langle u_{\mu} u_{v}\right\rangle\left\{D^{\mu}, D^{\nu}\right\} \psi+h . c .\right\} \\
& -\left\{\frac{c_{3}}{8 m^{2}} \bar{\psi}\left\{u_{\mu}, u_{v}\right\}\left\{D^{\mu}, D^{\nu}\right\} \psi+h . c .\right\}+\frac{c_{4}}{2} \bar{\psi}\left\langle u^{2}\right\rangle \psi \\
& +\frac{c_{5}}{2} \bar{\psi} u^{2} \psi+\left\{\frac{i c_{6}}{4} \bar{\psi} \sigma^{\mu \nu}\left[u_{\mu}, u_{\nu}\right] \psi+h . c .\right\}+c_{7} \bar{\psi} \hat{\chi}_{+} \psi \\
& +\frac{c_{8}}{8 m} \bar{\psi} \sigma^{\mu v} f_{\mu \nu}^{+} \psi+\frac{c_{9}}{8 m} \bar{\psi} \sigma^{\mu \nu}\left\langle f_{\mu \nu}^{+}\right\rangle \psi \\
\mathcal{L}^{(3)}= & \bar{\psi}\left\{\frac{h_{1}}{2} \gamma^{\mu} \gamma_{5}\left\langle\chi_{+}\right\rangle u_{\mu}+\frac{h_{2}}{2} \gamma^{\mu} \gamma_{5}\left\{\hat{\chi}_{+}, u_{\mu}\right\}+\frac{h_{3}}{2} \gamma^{\mu} \gamma_{5}\left\langle\hat{\chi}_{+} u_{\mu}\right\rangle\right. \\
& +\ldots\} \psi, \\
\mathcal{L}^{(4)}= & e_{1} \bar{\psi}\left\langle\chi_{+}\right\rangle\left\langle\chi_{+}\right\rangle \psi+e_{2} \bar{\psi} \hat{\chi}_{+}\left\langle\chi_{+}\right\rangle \psi+e_{3} \bar{\psi}\left\langle\hat{\chi}_{+} \hat{\chi}_{+}\right\rangle \psi \\
& +e_{4} \bar{\psi} \hat{\chi}+\hat{\chi}_{+} \psi+e_{5} \bar{\psi}\left\langle\chi_{-}\right\rangle\left\langle\chi_{-}\right\rangle \psi+e_{6} \bar{\psi} \hat{\chi}-\left\langle\chi_{-}\right\rangle \psi \\
& +e_{7} \bar{\psi}\left\langle\hat{\chi}_{-} \hat{\chi}_{-}\right\rangle \psi+e_{8} \bar{\psi} \hat{\chi}-\hat{\chi}_{-} \psi+\ldots .
\end{aligned}
$$

where $\hat{A}=A-\frac{1}{3}\langle A\rangle$ and $\langle A\rangle$ denotes the trace of $A$ in the flavor space. In the above Lagrangians, $c_{i}(i=1, \cdots, 9), h_{j}$ $(j=1, \cdots, 3)$, and $e_{k}(k=1, \cdots, 8)$ are the effective coupling constants. They are sometimes denoted as the low energy constants (LECs).

Since the doubly heavy baryons are very heavy, we can take the non-relativistic limit of the fully relativistic theory and expands the Lagrangian in power of the inverse of the doubly heavy baryon mass. The four-momentum of the doubly heavy baryon can be written as

$$
p_{\mu}=m v_{\mu}+l_{\mu}
$$

where $v_{\mu}$ is the four-velocity and $l_{\mu}$ the small off-shell momentum, which satisfies $v \cdot l \ll m$. The baryon field is decomposed into the light and heavy components $\psi=e^{-i m v \cdot x}(H+h)$, where $\psi H=H, w h=-h$.

The generating functional for the relativistic theory reads

$$
\begin{aligned}
\exp i Z[\eta, \bar{\eta}, v, a, s, p]= & \int[d \psi][d \bar{\psi}][d u] \exp \{i[S \\
& \left.\left.+\int d^{4} x(\bar{\eta} \psi+\bar{\psi} \eta)\right]\right\},
\end{aligned}
$$

where

$$
S=\int d^{4} x \mathcal{L} .
$$


In the terms of the fields $H$ and $h$, we can rewrite the original Lagrangian

$$
\mathcal{L}=\bar{H} \mathcal{A} H+\bar{h} \mathcal{B} H+\bar{H} \gamma^{0} \mathcal{B}^{\dagger} \gamma^{0} h-\bar{h} C h .
$$

$\mathcal{A}, \mathcal{B}$ and $C$ in Eq. (20) can be expanded in series of terms of different orders of $q^{i}$, where $q$ is the low energy momentum,

$$
\begin{aligned}
\mathcal{A} & =\mathcal{A}_{(1)}+\mathcal{A}_{(2)}+\ldots \\
\mathcal{B} & =\mathcal{B}_{(1)}+\mathcal{B}_{(2)}+\ldots \\
C & =C_{(1)}+C_{(2)}+\ldots
\end{aligned}
$$

The expressions of $\mathcal{A}, \mathcal{B}, C$ are collected in Appendix. With the replacement

$$
\begin{aligned}
& R=\frac{1}{2}(1+\not) e^{i m v \cdot x} \eta, \\
& \rho=\frac{1}{2}(1-\not) e^{i m v \cdot x} \eta,
\end{aligned}
$$

we have

$$
\bar{\eta} \psi+\bar{\psi} \eta=\bar{R} H+\bar{H} R+\bar{\rho} h+\bar{h} \rho .
$$

With $h^{\prime}=h-C^{-1}(\mathcal{B} H+\rho)$ and after integrating out the heavy degrees of freedom, the generating functional becomes

$$
\begin{aligned}
\exp i Z[R, \bar{R}, \rho, \bar{\rho}, v, a, s, p]= & \int[d H][d \bar{H}][d u] \Delta_{h} \exp i\left[S^{\prime}\right. \\
& \left.+\int d^{4} x(\bar{R} H+\bar{H} R)\right],
\end{aligned}
$$

where

$$
S^{\prime}=\int d^{4} x \bar{H}\left[\mathcal{A}+\left(\gamma_{0} \mathcal{B}^{\dagger} \gamma_{0}\right) C^{-1} \mathcal{B}\right] H
$$

and $\Delta_{h}$ is a constant. Then, one expands the matrix $C^{-1}$ in terms of $1 / m$

$$
\begin{aligned}
C^{-1}= & \frac{1}{2 m}-\frac{i(v \cdot D)+g_{A} S_{v} \cdot u}{(2 m)^{2}}-\frac{C_{(2)}}{(2 m)^{2}} \\
& +\frac{\left(i v \cdot D+g_{A} S_{v} \cdot u\right)^{2}}{(2 m)^{3}}+\cdots .
\end{aligned}
$$

Finally, the non-relativistic Lagrangians corresponding to the action $S^{\prime}$ can be expressed as

$$
\mathcal{L}^{\prime}=\mathcal{L}_{(1)}^{\prime}+\mathcal{L}_{(2)}^{\prime}+\mathcal{L}_{(3)}^{\prime}+\mathcal{L}_{(4)}^{\prime}+\cdots
$$

with $\mathcal{L}_{(i)}^{\prime}=\bar{H} T_{(i)} H(i=1,2,3,4, \cdots)$, where

$$
\begin{gathered}
T_{(1)}=i(v \cdot D)+g_{A} S_{v} \cdot u, \\
T_{(2)=} c_{1}\left\langle\chi_{+}\right\rangle+\frac{c_{2}}{2}\left\langle(v \cdot u)^{2}\right\rangle+c_{3}(v \cdot u)^{2}+\frac{c_{4}}{2}\left\langle u^{2}\right\rangle \\
+\frac{c_{5}}{2} u^{2}+\frac{c_{6}}{2}\left[S_{v}^{\mu}, S_{v}^{v}\right]\left[u_{\mu}, u_{v}\right]+c_{7} \hat{\chi_{+}} \\
-\frac{i c_{8}}{4 m}\left[S_{v}^{\mu}, S_{v}^{v}\right] f_{\mu v}^{+}-\frac{i c_{9}}{4 m}\left[S_{v}^{\mu}, S_{v}^{v}\right]\left\langle f_{\mu v}^{+}\right\rangle
\end{gathered}
$$

$$
\begin{aligned}
& +\frac{2}{m}\left(S_{v} \cdot D\right)^{2}-\frac{i g_{A}}{2 m}\left\{S_{v} \cdot D, v \cdot u\right\} \\
& -\frac{g_{A}^{2}}{8 m}(v \cdot u)^{2}+\ldots,
\end{aligned}
$$

$$
\begin{aligned}
T_{(3)}= & h_{1} S_{v}^{\mu}\left\langle\chi_{+}\right\rangle u_{\mu}+h_{2} S_{v}^{\mu}\left\{\hat{\chi}_{+}, u_{\mu}\right\}+h_{3} S_{v}^{\mu}\left\langle\hat{\chi}_{+} u_{\mu}\right\rangle \\
& -\frac{c_{8}}{4 m^{2}} S_{v} \cdot D\left(v^{\mu} S_{v}^{v}-v^{\nu} S_{v}^{\mu}\right) f_{\mu v}^{+} \\
& -\frac{c_{9}}{4 m^{2}} S_{v} \cdot D\left(v^{\mu} S_{v}^{v}-v^{\nu} S_{v}^{\mu}\right)\left\langle f_{\mu v}^{+}\right\rangle \\
& +\frac{i g_{A} c_{8}}{16 m^{2}}\left(v^{\mu} S_{v}^{v}-v^{v} S_{v}^{\mu}\right)\left[v \cdot u, f_{\mu v}^{+}\right] \\
& \frac{c_{8}}{4 m^{2}}\left(v^{\mu} S_{v}^{v}-v^{v} S_{v}^{\mu}\right) f_{\mu v}^{+} S_{v} \cdot D \\
& +\frac{c_{9}}{4 m^{2}}\left(v^{\mu} S_{v}^{v}-v^{\nu} S_{v}^{\mu}\right)\left\langle f_{\mu \nu}^{+}\right\rangle S_{v} \cdot D \\
& -\frac{i}{m^{2}} S_{v} \cdot D v \cdot D S_{v} \cdot D+\ldots,
\end{aligned}
$$

$$
\begin{aligned}
& T_{(4)}=e_{1}\left\langle\chi_{+}\right\rangle\left\langle\chi_{+}\right\rangle+e_{2} \hat{\chi_{+}}\left\langle\chi_{+}\right\rangle+e_{3}\left\langle\hat{\chi_{+}} \hat{\chi_{+}}\right\rangle+e_{4} \hat{\chi_{+}} \hat{\chi_{+}} \\
& \left.+e_{5}\left\langle\chi_{-}\right\rangle\left\langle\chi_{-}\right\rangle+e_{6} \hat{\chi_{-}}\left\langle\chi_{-}\right\rangle+e_{7} \hat{\chi_{-}} \hat{\chi_{-}}\right\rangle+e_{8} \hat{\chi_{-}} \hat{\chi_{-}} \\
& -\frac{c_{8}^{2}}{32 m^{3}}\left(v^{\mu} S_{v}^{v}-v^{v} S_{v}^{\mu}\right) f_{\mu \nu}^{+}\left(v^{\alpha} S_{v}^{\beta}-v^{\beta} S_{v}^{\alpha}\right) f_{\alpha \beta}^{+} \\
& -\frac{c_{8} c_{9}}{32 m^{3}}\left(v^{\mu} S_{v}^{v}-v^{v} S_{v}^{\mu}\right) f_{\mu v}^{+}\left(v^{\alpha} S_{v}^{\beta}-v^{\beta} S_{v}^{\alpha}\right)\left\langle f_{\alpha \beta}^{+}\right\rangle \\
& -\frac{c_{8} c_{9}}{32 m^{3}}\left(v^{\mu} S_{v}^{v}-v^{v} S_{v}^{\mu}\right)\left\langle f_{\mu \nu}^{+}\right\rangle\left(v^{\alpha} S_{v}^{\beta}-v^{\beta} S_{v}^{\alpha}\right) f_{\alpha \beta}^{+} \\
& -\frac{c_{9}^{2}}{32 m^{3}}\left(v^{\mu} S_{v}^{v}-v^{v} S_{v}^{\mu}\right)\left\langle f_{\mu v}^{+}\right\rangle\left(v^{\alpha} S_{v}^{\beta}-v^{\beta} S_{v}^{\alpha}\right)\left\langle f_{\alpha \beta}^{+}\right\rangle \\
& +\frac{i c_{8}}{(2 m)^{3}} S_{v} \cdot D v \cdot D\left(v^{\mu} S_{v}^{v}-v^{v} S_{v}^{\mu}\right) f_{\mu v}^{+} \\
& +\frac{i c_{9}}{(2 m)^{3}} S_{v} \cdot D v \cdot D\left(v^{\mu} S_{v}^{v}-v^{v} S_{v}^{\mu}\right)\left\langle f_{\mu \nu}^{+}\right\rangle \\
& -\frac{i c_{8}}{(2 m)^{3}}\left(v^{\mu} S_{v}^{v}-v^{v} S_{v}^{\mu}\right) f_{\mu \nu}^{+} v \cdot D S_{v} \cdot D \\
& -\frac{i c_{9}}{(2 m)^{3}}\left(v^{\mu} S_{v}^{v}-v^{v} S_{v}^{\mu}\right)\left\langle f_{\mu \nu}^{+}\right\rangle v \cdot D S_{v} \cdot D \\
& +\frac{c_{7}}{m^{2}} S_{v} \cdot D \hat{\chi_{+}} S_{v} \cdot D+\frac{c_{1}}{m^{2}} S_{v} \cdot D\left\langle\chi_{+}\right\rangle S_{v} \cdot D \\
& -\frac{i c_{8}}{4 m^{3}} S_{v} \cdot D\left[S_{v}^{\mu}, S_{v}^{v}\right] f_{\mu v}^{+} S_{v} \cdot D \\
& -\frac{i c_{9}}{4 m^{3}} S_{v} \cdot D\left[S_{v}^{\mu}, S_{v}^{v}\right]\left\langle f_{\mu \nu}^{+}\right\rangle S_{v} \cdot D \\
& -\frac{1}{2 m^{3}} S_{v} \cdot D(v \cdot D)^{2} S_{v} \cdot D+\ldots,
\end{aligned}
$$

where

$$
S_{v}^{\mu}=\frac{i}{2} \gamma_{5} \sigma^{\mu v} v_{v}
$$

The above Lagrangians will be employed to calculate the chiral correction to the mass and axial current of the doubly charmed baryons. 


\section{THE CHIRAL CORRECTION TO THE MASS OF THE DOUBLY HEAVY BARYON}

With the notations $\eta=v \cdot p$ and $\xi=(p-m v)^{2}$, the full propagator of the doubly heavy baryon is written as

$$
\begin{aligned}
G & =\frac{i}{v \cdot p-m_{0}-\Sigma_{B}(\eta, \xi)} \\
& =\frac{i Z_{N}}{v \cdot p-m-Z_{N} \tilde{\Sigma}_{B}(\eta, \xi)}
\end{aligned}
$$

where $\Sigma_{B}(\eta, \xi)$ denotes the high order contributions to the selfenergy, which are from Fig. 1 (a-h).
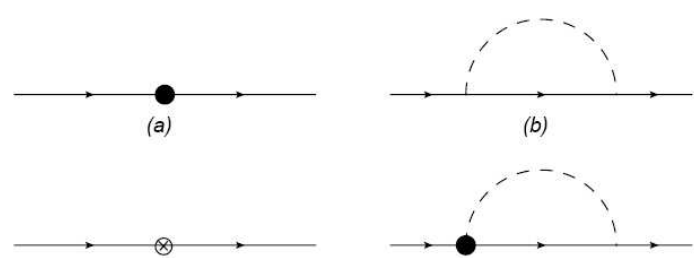

(c)

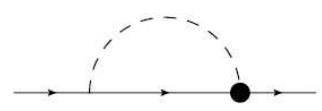

(e)

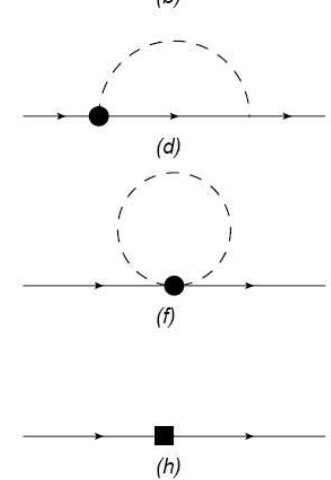

(g)

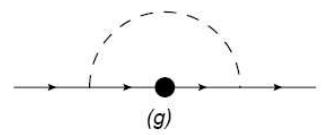

(h)

FIG. 1: The Feynman diagrams which contribute to the self-energy of doubly charmed baryon. The solid and dashed lines denote the doubly charmed baryons and goldstone bosons. The solid dot, circlecross and black box denote the vertices from the $O\left(p^{2}, p^{3}, p^{4}\right)$ Lagrangians respectively.

The mass of the doubly charmed baryon is

$$
m=m_{0}+\Sigma_{B}(0,0) \text {. }
$$

And the renormalization constant reads

$$
Z_{N}=\frac{1}{1-\Sigma_{B}^{\prime}(0,0)}
$$

with

$$
\Sigma_{B}^{\prime}(0,0)=\left.\frac{\partial \Sigma_{B}(\eta, \xi)}{\partial \eta}\right|_{(\eta, \xi)=(0,0)}
$$

The chiral contribution to the self-energy up to next-to-nextto-leading order (NNLO) includes three pieces

$$
\begin{aligned}
\Sigma_{B}^{(a)} & =-\left\{2 c_{1}\langle\chi\rangle+2 c_{7} \hat{\chi}_{i i}-\frac{2}{m} S_{v}^{\mu} S_{v}^{v} k_{\mu} k_{v}\right\}, \\
\Sigma_{B, P}^{(b)} & =i C_{B P}^{(b)} \int \frac{d^{4} q}{(2 \pi)^{4}}\left[\frac{g_{A}}{F_{P 0}} S_{v} \cdot q\right] \frac{i}{v \cdot(k-q)+i \epsilon}
\end{aligned}
$$

$$
\begin{aligned}
& \times \frac{i}{q^{2}-M_{P}^{2}+i \epsilon}\left[-\frac{g_{A}}{F_{P 0}} S_{v} \cdot q\right] \\
= & -C_{B P}^{(b)} \frac{g_{A}^{2}}{\left(4 \pi F_{P 0}\right)^{2}}\left\{\frac { v \cdot k _ { u } } { 4 } \left(\left[3 M_{P}^{2}-2\left(v \cdot k_{u}\right)^{2}\right][R\right.\right. \\
& \left.\left.+\ln \left(\frac{M_{P}^{2}}{\mu^{2}}\right)\right]-2\left[M_{P}^{2}-\left(v \cdot k_{u}\right)^{2}\right]\right) \\
& \left.+\left[M_{P}^{2}-\left(v \cdot k_{u}\right)^{2}\right]^{3 / 2} \arccos \left(-\frac{v \cdot k_{u}}{M_{P}}\right)\right\} \\
\Sigma_{B}^{(c)}= & -\frac{1}{m^{2}}\left(S_{v} \cdot k\right)(v \cdot k)\left(S_{v} \cdot k\right),
\end{aligned}
$$

which correspond to Fig. 1 (a)-(c), respectively.

$$
\Sigma_{B}=\Sigma_{B}^{(a)}+\sum_{P} \Sigma_{B, P}^{(b)}+\Sigma_{B}^{(c)}
$$

with the subscripts $B=\Xi_{c c}^{++}, \Xi_{c c}^{+}, \Omega_{c c}^{+}$and $P=$ $\pi^{ \pm, 0}, K^{ \pm, 0}, \bar{K}^{0}, \eta . \quad F_{\pi 0}, F_{K 0}$ and $F_{\eta 0}$ are the decay constants of $\pi, K$ and $\eta$, which are $0.092,0.112$ and $0.110 \mathrm{GeV}$, respectively. In addition, the coefficients $C_{B P}^{(b)}$ are given in Table II.

We notice that there are three low energy constants $c_{1}, c_{7}$, and $g_{A}$ in Eqs. (38)-(40). Among these LECs, $c_{1}$ appearing in the next-to-leading order Lagrangian can be absorbed into the bare mass term. Thus, $c_{7}$ and $g_{A}$ are the two unknown constant. Due to the absence of the corresponding experimental information, we have to fix these unknown constants based on the other theoretical calculations. In Ref. [64], Hu and Mehen constructed Lagrangian with the following form

$$
\mathcal{L}=\operatorname{Tr}\left[T_{a}^{\dagger}\left(i D_{0}\right)_{b a} T_{b}\right]-g \operatorname{Tr}\left[T_{a}^{\dagger} T_{b} \vec{\sigma} \cdot \vec{A}_{b a}\right]+\ldots
$$

by considering the heavy diquark symmetry, where $T_{a, i \beta}=$ $\sqrt{2}\left(\Xi_{a, i \beta}^{*}+\frac{1}{\sqrt{3}} \Xi_{a, \gamma} \sigma_{\gamma \beta}^{i}\right)$. In Eq. (42), the coupling $g=0.6$ is determined by fitting the $D^{*+}$ width. Comparing our effective Lagrangian with that in Eq. (42), we get $g_{A}=g$. In the following, we take $g_{A}=0.6$.

The LEC $c_{7}$ and bare mass $m_{0}$ are still unknown. We try to fix these two unknown constants by fitting the lattice data with pion mass upto $0.4 \mathrm{GeV}$ in Ref. [27].

The masses of $\Xi_{c c}$ are given for different $m_{\pi}$ and $m_{c}$ in Ref. [27]. We assume only the bare mass $\left(m_{0}\right)$ depends on the mass $\left(m_{c}\right)$ of the valence charm quark, and the dependence respects the heavy quark expansion

$$
m_{0}=\tilde{m}_{0}+2 m_{c}+\alpha / m_{c}+O\left(1 / m_{c}^{2}\right) .
$$

The physical mass $\left.m_{c}\right|_{\text {phy }}$ is tuned to reproduce the mass of $D$ meson at the physical point in Ref. [27]

$$
\left.m_{c}\right|_{\text {phy }}=0.591 \pm 0.028 \mathrm{GeV} \text {. }
$$

We give the fitted results with $\chi_{\text {dof }}^{2} \lesssim 1$ in Table III. Generally speaking, it indicates the lattice data are over-fitted if a result with $\chi_{\text {dof }}^{2} \lesssim 1$ could be obtained. One can fit the lattice data well with any $c_{7}$ lying in the range $(-6.0,0.6)$ from the table. Therefore the current lattice data of $m_{\Xi_{c c}}$ are not enough to constrain $c_{7}$ yet. However, $c_{7}$ being around -0.2 might be 


\begin{tabular}{c|cccccccc}
\hline$\left(C_{B P}^{(b / d / g)}\right)^{1 / 2}$ & $\pi^{+}$ & $\pi^{0}$ & $\pi^{-}$ & $K^{+}$ & $K^{0}$ & $\bar{K}^{0}$ & $K^{-}$ & $\eta$ \\
\hline$\Xi_{c c}^{++}$ & $\sqrt{2}$ & 1 & 0 & $\sqrt{2}$ & 0 & 0 & 0 & $\frac{1}{\sqrt{3}}$ \\
$\Xi_{c c}^{+}$ & 0 & -1 & $\sqrt{2}$ & 0 & $\sqrt{2}$ & 0 & 0 & $\frac{1}{\sqrt{3}}$ \\
$\Omega_{c c}^{+}$ & 0 & 0 & 0 & 0 & 0 & $\sqrt{2}$ & $\sqrt{2}$ & $-\frac{2}{\sqrt{3}}$ \\
\hline$\left(C_{1 B P}^{(f)}\right)^{1 / 2}$ & $\pi^{+}$ & $\pi^{0}$ & $\pi^{-}$ & $K^{+}$ & $K^{0}$ & $\bar{K}^{0}$ & $K^{-}$ & $\eta$ \\
\hline$\Xi_{c c}^{++}$ & $\sqrt{2}$ & 1 & 0 & $\sqrt{2}$ & 0 & 0 & 0 & $\frac{1}{\sqrt{3}}$ \\
$\Xi_{c c}^{+}$ & 0 & -1 & $\sqrt{2}$ & 0 & $\sqrt{2}$ & 0 & 0 & $\frac{1}{\sqrt{3}}$ \\
$\Omega_{c c}^{+}$ & 0 & 0 & 0 & 0 & 0 & $\sqrt{2}$ & $\sqrt{2}$ & $-\frac{2}{\sqrt{3}}$ \\
\hline$C_{2 B P}^{(f)}$ & $\pi^{+}$ & $\pi^{0}$ & $\pi^{-}$ & $K^{+}$ & $K^{0}$ & $\bar{K}^{0}$ & $K^{-}$ & $\eta$ \\
\hline$\Xi_{c c}^{++} / \Xi_{c c}^{+} / \Omega_{c c}^{+}$ & 2 & 2 & 2 & 2 & 2 & 2 & 2 & 2 \\
\hline$C_{3 B P}^{(f)}$ & $\pi^{+}$ & $\pi^{0}$ & $\pi^{-}$ & $K^{+}$ & $K^{0}$ & $\bar{K}^{0}$ & $K^{-}$ & $\eta$ \\
\hline$\Xi_{c c}^{++}$ & $4 B_{0} m_{d}$ & $2 B_{0}\left(m_{u}\right)$ & 0 & $4 B_{0} m_{s}$ & 0 & 0 & 0 & $\frac{2}{3} B_{0}\left(m_{u}\right)$ \\
$\Xi_{c c}^{+}$ & 0 & $2 B_{0}\left(m_{d}\right)$ & $4 B_{0} m_{u}$ & 0 & 0 & 0 & $\frac{2}{3} B_{0}\left(m_{d}\right)$ & \\
$\Omega_{c c}^{+}$ & 0 & 0 & 0 & 0 & 0 & $4 B_{0}\left(m_{d}\right)$ & $4 B_{0}\left(m_{u}\right)$ & $\frac{8}{3} B_{0}\left(m_{s}\right)$ \\
\hline$C_{4 B P}^{(f)}$ & $\pi^{+}$ & $\pi^{0}$ & $\pi^{-}$ & $K^{+}$ & $K^{0}$ & $\bar{K}^{0}$ & $K^{-}$ & $\eta$ \\
\hline$\Xi_{c c}^{++}$ & $4 B_{0} m_{u}$ & $2 B_{0}\left(m_{u}\right)$ & 0 & $4 B_{0} m_{u}$ & 0 & 0 & 0 & $\frac{2}{3} B_{0}\left(m_{u}\right)$ \\
$\Xi_{c c}^{+}$ & 0 & $2 B_{0}\left(m_{d}\right)$ & $4 B_{0} m_{d}$ & 0 & $4 B_{0}\left(m_{d}\right)$ & 0 & 0 & $\frac{2}{3} B_{0}\left(m_{d}\right)$ \\
$\Omega_{c c}^{+}$ & 0 & 0 & 0 & 0 & 0 & $4 B_{0}\left(m_{s}\right)$ & $4 B_{0}\left(m_{s}\right)$ & $\frac{8}{3} B_{0}\left(m_{s}\right)$ \\
\hline$C_{5 B P}^{(f)}$ & $\pi^{+}$ & $\pi^{0}$ & $\pi^{-}$ & $K^{+}$ & $K^{0}$ & $\bar{K}^{0}$ & $K^{-}$ & $\eta$ \\
\hline$\Xi_{c c}^{++} / \Xi_{c c}^{+} / \Omega_{c c}^{+}$ & $4 B_{0} m_{u}$ & $2 B_{0}\left(m_{u}+m_{d}\right)$ & $4 B_{0} m_{d}$ & $4 B_{0} m_{u}$ & $4 B_{0} m_{d}$ & $4 B_{0} m_{s}$ & $4 B_{0} m_{s}$ & $\frac{2}{3} B_{0}\left(m_{u}+m_{d}+4 m_{s}\right)$ \\
\hline
\end{tabular}

TABLE II: The values of the coefficients $\left(C_{B P}^{(b / d / g)}\right)^{1 / 2},\left(C_{1 B P}^{(f)}\right)^{1 / 2}, C_{2 B P}^{(f)}, C_{3 B P}^{(f)}, C_{4 B P}^{(f)}$, and $C_{5 B P}^{(f)}$ in Eqs. (38)-(40) and (55)-(60).

TABLE III: Parameters for fitting the lattice data from Ref. [27] and the physical masses of doubly charmed baryons with the corresponding fitted parameters. The error of the masses are from the error of $\left.m_{c}\right|_{\text {phy }}$.

\begin{tabular}{rcc|c|cc}
\hline$c_{7}$ & $\tilde{m}_{0}$ & $\alpha$ & $\chi_{\text {dof }}^{2}$ & $m_{\Xi_{c c}}$ & $m_{\Omega_{c c}}$ \\
\hline 0.6 & 3.314 & -0.518 & 1.0 & $3.710_{-.100}^{+.096}$ & $3.045_{-.100}^{+.096}$ \\
0.3 & 3.363 & -0.505 & 0.8 & $3.690_{-.099}^{+.095}$ & $3.297_{-.099}^{+.095}$ \\
0.0 & 3.450 & -0.510 & 0.7 & $3.677_{-.099}^{+.095}$ & $3.557_{-.099}^{+.095}$ \\
-0.1 & 3.472 & -0.509 & 0.6 & $3.672_{-.099}^{+.095}$ & $3.642_{-.099}^{+.095}$ \\
-0.2 & 3.460 & -0.488 & 0.6 & $3.665_{-.097}^{+.093}$ & $3.726_{-.097}^{+.093}$ \\
-0.3 & 3.517 & -0.506 & 0.5 & $3.661_{-.099}^{+.095}$ & $3.813_{-.099}^{+.095}$ \\
-0.4 & 3.541 & -0.506 & 0.5 & $3.655_{-.099}^{+.095}$ & $3.898_{-.099}^{+.095}$ \\
-0.5 & 3.562 & -0.503 & 0.4 & $3.650_{-.098}^{+.095}$ & $3.983_{-.098}^{+.095}$ \\
-1.0 & 3.552 & -0.427 & 0.4 & $3.618_{-.092}^{+.089}$ & $4.405_{-.092}^{+.089}$ \\
-2.0 & 3.900 & -0.484 & 0.1 & $3.567_{-.097}^{+.093}$ & $5.261_{-.097}^{+.093}$ \\
-4.0 & 4.351 & -0.458 & 0.4 & $3.457_{-.095}^{+.091}$ & $6.966_{-.095}^{+.091}$ \\
-6.0 & 4.801 & -0.431 & 1.6 & $3.347_{-.092}^{+.089}$ & $8.671_{-.092}^{+.089}$ \\
\hline
\end{tabular}

a real solution considering that the mass of $\Omega_{c c}$ is $3.68 \sim 3.76$ GeV by lattice QCD groups [23-27].

We plot the best fitted results with supposing $c_{7}=-0.2$ in
Fig. 2. The best fitting needs $\tilde{m}_{0}=3.460$ and $\alpha=-0.488$ and predicts

$$
m_{\Xi_{c c}}=3.665_{-.097}^{+.093} \mathrm{GeV}, \quad m_{\Omega_{c c}}=3.726_{-.097}^{+.093} \mathrm{GeV} .
$$

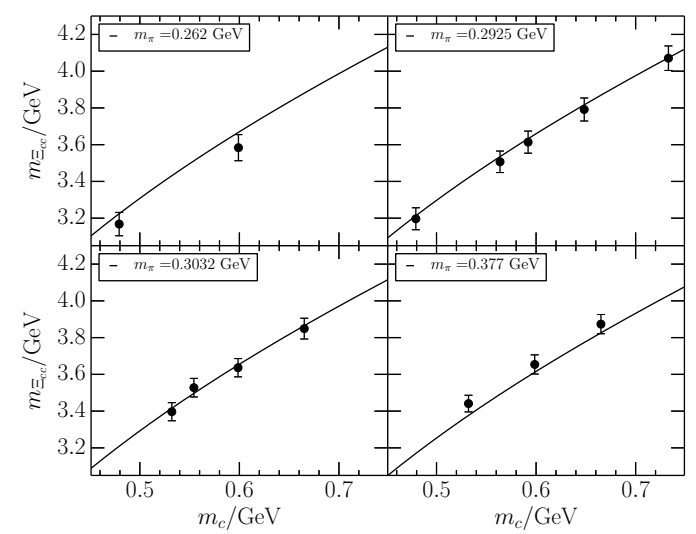

FIG. 2: The masses of $\Xi_{c c}$ as a function of $m_{c}$ for different masses of pion. The lattice data are from Ref. [27], and the solid curves are our fitted results upto next to the leading order with $c_{7}=-0.2$, $\tilde{m}_{0}=3.460, \alpha=-0.488$, and $\chi_{\text {dof }}^{2}=0.6$

We have also obtained the mass correction of the doubly charmed baryon up to the next-next-next-Leading order 
$\left(\mathrm{N}^{3} \mathrm{LO}\right)$, which is collected in the Appendix A. Unfortunately there appear too many unknown LECs which cannot be fixed by experimental or theoretical approaches. We are unable to use the $\mathrm{N}^{3} \mathrm{LO}$ mass formula to compare with the current experimental data. However, the chiral structure and expression of the mass at the $\mathrm{N}^{3} \mathrm{LO}$ will be helpful to the chiral extrapolation of the lattice data in the lattice QCD simulation.

\section{THE CHIRAL CORRECTION TO THE AXIAL CURRENT}

In the following, we discuss the chiral correction to the axial current of the doubly charmed baryon. Using Lagrangian $\mathcal{L}_{(1)}^{\prime}$ and $\mathcal{L}_{(3)}^{\prime}$ in Eq. (55), we obtain the axial current at the tree level

$$
\begin{aligned}
A^{k, \mu}= & \frac{\partial \mathcal{L}}{\partial r_{k}^{\mu}}-\frac{\partial \mathcal{L}}{\partial l_{k}^{\mu}} \\
= & \frac{1}{2} v^{\mu} \bar{H}\left(u^{\dagger} T^{k} u-u T^{k} u^{\dagger}\right) H \\
& +g_{A} \bar{H} S_{v}^{\mu}\left(u^{\dagger} T^{k} u+u T^{k} u^{\dagger}\right) H \\
& +h_{1} \bar{H} S_{v}^{\mu}\left\langle\chi_{+}\right\rangle\left(u^{\dagger} T^{k} u+u T^{k} u^{\dagger}\right) H \\
& +h_{2} \bar{H} S_{v}^{\mu}\left\{\hat{\chi}_{+},\left(u^{\dagger} T^{k} u+u T^{k} u^{\dagger}\right)\right\} H \\
& +h_{3} \bar{H} S_{v}^{\mu}\left\langle\hat{\chi}_{+}\left(u^{\dagger} T^{k} u+u T^{k} u^{\dagger}\right)\right\rangle H .
\end{aligned}
$$

We collect the diagrams contributing to the renormalization of the axial currents in Fig. 3.

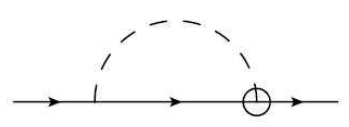

(1)

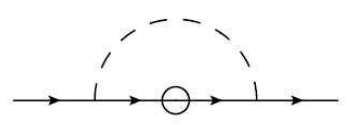

(3)

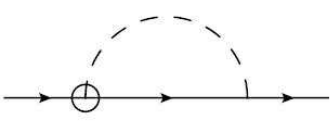

(2)

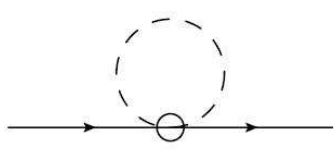

(4)
FIG. 3: The diagrams contributing to the renormalization of the axial current. The circle represents an insertion of the axial current.

The renormalized matrix element of $A^{k, \mu}$ between the doubly heavy baryon states can be written as

$$
\begin{aligned}
\left\langle B_{d}\left|A^{k, \mu}\right| B_{a}\right\rangle & \left\{g _ { A } 2 T _ { a d } ^ { k } \left[1-\sum_{P} \frac{g_{A}^{2}}{\left(4 \pi F_{P 0}\right)^{2}}\left(\frac{3 M_{P}^{2}}{4}\left(R+\ln \frac{M_{P}^{2}}{\mu^{2}}\right)\right.\right.\right. \\
& \left.\left.+\frac{1}{2} M_{P}^{2}\right)\left(C_{B_{a} P}^{(b)}+C_{B_{d} P}^{(b)}\right) \frac{1}{2}\right]+h_{1}\left\langle\chi_{+}\right\rangle 2 T_{a d}^{k} \\
& +h_{2} \sum_{i}\left[2 \chi_{a i} T_{i d}^{k}+2 T_{a i}^{k} \chi_{i d}\right]+h_{3} 2\left\langle\chi_{+} T^{k}\right\rangle \\
& \left.+\sum_{P, b, c} \Sigma_{b c}^{\text {current }}\right\} \bar{u}_{a} S_{v}^{\mu} u_{d} .
\end{aligned}
$$

In the above equations, we have

$$
\Sigma^{\text {current }}=\Sigma_{(1)}^{\text {current }}+\Sigma_{(2)}^{\text {current }}+\Sigma_{(3)}^{\text {current }}+\Sigma_{(4)}^{\text {current }},
$$

where

$$
\Sigma_{(1)}^{\text {current }}=0,
$$

$$
\Sigma_{(2)}^{\text {current }}=0
$$

$$
\begin{aligned}
\Sigma_{(3)}^{\text {current }}= & -\frac{g_{A}^{3}}{6 F_{P 0}^{2}} C_{\tilde{P} a b} \delta_{c d} \delta_{a b} T_{b c}^{k}\left\{-2(v \cdot k)\left[-4 \frac{1}{32 \pi^{2}}\left(R-\frac{2}{3}\right)\right.\right. \\
& \times \frac{v \cdot k}{8 \pi^{2}}\left(1-2 \ln \frac{M_{\tilde{P}}}{\mu}\right)-\frac{1}{4 \pi^{2}} \sqrt{M_{\tilde{P}}^{2}-(v \cdot k)^{2}} \\
& \left.\times \arccos \frac{-v \cdot k}{M_{\tilde{P}}}\right]+\left[M_{\tilde{P}}^{2}-(v \cdot k)^{2}\right]\left[-4 \frac{1}{32 \pi^{2}}\left(R-\frac{2}{3}\right)\right. \\
& +\frac{1}{8 \pi^{2}}\left(1-2 \ln \frac{M_{\tilde{P}}}{\mu}\right)+\frac{v \cdot k}{2 \pi^{2}}\left[M_{\tilde{P}}^{2}-(v \cdot k)^{2}\right]^{-1 / 2} \\
& \left.\times \arccos \frac{-v \cdot k}{M_{\tilde{P}}}-\frac{1}{4 \pi^{2}}\right]-2 M_{\tilde{P}}^{2}\left(\frac{1}{32 \pi^{2}}\left(R-\frac{2}{3}\right)\right. \\
& \left.\left.+\frac{1}{16 \pi^{2}} \ln \frac{M_{\tilde{P}}}{\mu}\right)\right\}, \\
\Sigma_{(4)}^{c u r r e n t} & =-\frac{i g_{A}}{4 F_{P 0}^{2}}\left[-C_{\tilde{P} b c} T_{a b}^{k} \delta_{b d}+2 C_{\tilde{P} a b} T_{b c}^{k} \delta_{a d} \delta_{b c}\right. \\
& \left.\quad-T_{c d}^{k} C_{\tilde{P} a b} \delta_{a c}\right] \frac{M_{\tilde{P}}^{2}}{(4 \pi)^{2}}\left[-R+\ln \frac{\mu^{2}}{M_{\tilde{P}}^{2}}\right]
\end{aligned}
$$

which correspond to Fig. 3 (1)-(4), respectively. Since the doubly heavy baryons are very heavy, we do not take into account the small recoil corrections in the present work. The contribution from Fig. 3 (1)-(2) vanishes as shown in Eqs. (48)-(49). In the above equations, the matrices $C_{\tilde{P}}(\tilde{P}=$ $\pi, K, \eta)$ are defined as

$$
C_{\pi}=\left(\begin{array}{lll}
1 & 2 & 0 \\
2 & 1 & 0 \\
0 & 0 & 0
\end{array}\right), C_{K}=\left(\begin{array}{lll}
0 & 0 & 2 \\
0 & 0 & 2 \\
2 & 2 & 0
\end{array}\right), C_{\eta}=\left(\begin{array}{ccc}
\frac{1}{3} & 0 & 0 \\
0 & \frac{1}{3} & 0 \\
0 & 0 & \frac{4}{3}
\end{array}\right) .
$$

The wave function renormalization constant is expressed as

$$
Z_{N, B P}=1-C_{B P}^{(b)} \frac{g_{A}^{2}}{\left(4 \pi F_{P 0}\right)^{2}}\left\{\frac{3 M_{P}^{2}}{4}\left(R+\ln \frac{M_{P}^{2}}{\mu^{2}}\right)+\frac{1}{2} M_{P}^{2}\right\} .
$$

We define the axial charge of the heavy baryon through the matrix element

$$
\left\langle B_{d}\left|A^{k, \mu}\right| B_{a}\right\rangle=g_{a d}^{A} \bar{u}_{d} S_{v}^{\mu} u_{a},
$$

where $g_{a d}^{A}$ is the axial charge.

In Eq. (47), there exist three low energy constants $h_{1}, h_{2}$ and $h_{3}$. The LEC $h_{1}$ can be absorbed into the $g_{A}$ term. There 
remain two unknown constants $h_{2}$ and $h_{3}$. At present, there is no enough information to fix $h_{2}$ and $h_{3}$. As a crude approximation, we simply parameterize $h_{2}$ and $h_{3}$ as $h_{2}=h_{3}=\frac{1}{\lambda^{2}}$, where $\lambda$ is the typical energy scale around the mass of the doubly heavy baryons. Taking the typical value $\lambda= \pm 3.6 \mathrm{GeV}$, we obtain $g_{\Xi_{c c}^{++} \Xi_{c c}^{+}}^{1+i 2}=1.15$ and $g_{\Xi_{c c}^{++} \Omega_{c c}^{+}}^{4+i 5}=1.18$. If only considering the tree level contribution, we get $g_{\Xi_{c c}^{++} \Xi_{c c}^{+}}^{1+i 2}=g_{\Xi_{c c}^{++} \Omega_{c c}^{+}}^{4+i 5}=1.2$. If $\lambda$ varies from 2 to $5 \mathrm{GeV}$, the range of $g_{\Xi_{c c}^{++} \Xi_{c c}^{+}}^{1+i 2}$ and $g_{\Xi_{c c}^{++} \Omega_{c c}^{+}}^{4+i 5}$ will be $1.16 \sim 1.14$ and $1.35 \sim 1.14$ respectively. We also consider the case $h_{2,3} \sim-\frac{1}{\lambda^{2}}$. And $g_{\Xi_{c c}^{++} \Xi_{c c}^{+}}^{1+i 2}$ and $g_{\Xi_{c c}^{++} \Omega_{c c}^{+}}^{4+i 5}$ will be $1.12 \sim 1.14$ and $0.86 \sim 1.07$ respectively, when $\lambda$ is in the range $2 \sim 5 \mathrm{GeV}$.

\section{SUMMARY}

Although the doubly heavy baryons have not been established experimentally, these systems are particularly interesting. To a large extent, they are even simpler than the light baryons such as nucleons where the interaction among the three light quarks is very complicated. In contrast, the presence of the two heavy quarks acts as a static color source in the heavy quark limit. For example, the chiral dynamics of the doubly heavy baryons is solely governed by the light quark. We can gain valuable insights into the light quark chiral behavior through the chiral perturbation theory study of the doubly heavy baryons.

In this work, we have constructed the chiral effective Lagrangians describing the interactions of light mesons and doubly charmed baryons. We further make the non-relativistic reduction and obtain the chiral Lagrangians up to $O\left(p^{4}\right)$ in the heavy baryon limit. We have derived the chiral corrections to the mass of the doubly heavy baryons up to $\mathrm{N}^{3} \mathrm{LO}$. Unfortunately there exist too many unknown low energy constants. We are forced to perform the numerical analysis at the $\mathrm{N}^{2} \mathrm{LO}$. The mass splitting of $\Xi_{c c}$ and $\Omega_{c c}$ at the NNLO depends on one unknown low energy constant $c_{7}$. By fitting the lattice data for $\Xi_{c c}$ from Ref. [27] and supposing $c_{7}=-0.2$, we estimate the mass of $\Omega_{c c}$ to be around $3.726 \mathrm{GeV}$, which may be helpful to further experimental search of the doubly charmed baryon $\Omega_{c c}$.

Moreover, we have also performed a systematical analysis of the chiral corrections to the axial currents and axial charges of the doubly heavy baryons, which may be measured through the semileptonic decays of the heavy baryons in the future.

The chiral corrections to the mass of the doubly heavy baryons have been derived up to $\mathrm{N}^{3} \mathrm{LO}$ and the axial charge to $\mathrm{N}^{2} \mathrm{LO}$. The chiral structure and analytical expressions will be very useful to the chiral extrapolations of the future lattice QCD simulations of the doubly heavy baryons.

The exploration of the doubly charmed baryons is still an important and intriguing research topic, which can deepen our understanding of hadron spectrum and nonperturbative QCD. We are looking forward to more developments from both experimental and theoretical studies. There is very good chance that these doubly charmed baryons will be observed at facilities such as LHC and BelleII.

\section{Acknowledgement}

We would like to thank Xiu-Lei Ren, Nan Jiang and all the members of "the workshop of chiral effective field theory2014" that held in Sichuan University. This project is supported by the National Natural Science Foundation of China under Grants No. 11222547, No. 11175073, No. 11035006, No. 11375240 and No. 11261130311, the Ministry of Education of China (FANEDD under Grant No. 200924, SRFDP under Grant No. 2012021111000, and NCET), the China Postdoctoral Science Foundation under Grant No. 2013M530461, and the Fok Ying Tung Education Foundation (Grant No. 131006). This work was also supported by the University of Adelaide and the Australian Research Council grant FL0992247.

\section{Appendix A: The $\mathrm{N}^{3} \mathrm{LO}$ contribution to the mass of the doubly charmed baryon}

We list the $\mathrm{N}^{3} \mathrm{LO}$ chiral corrections to the mass of the doubly charmed baryon, i.e.,

$$
\begin{aligned}
\Sigma_{B, P}^{(d)}= & C_{B P}^{(d)} i \int \frac{d^{4} q}{(2 \pi)^{4}}(-) \frac{g_{A}}{2 m F_{0}} S_{v}^{\mu} v^{v}\left(-q_{\mu} q_{v}+2 q_{v} k_{\mu}\right) \\
& \times \frac{i}{q^{2}-M^{2}+i \epsilon} \frac{i}{v \cdot(k-q)+i \epsilon}(-) \frac{g_{A}}{F_{0}} S_{v}^{\alpha} q_{\alpha} \\
= & C_{B P}^{(d)} \frac{g_{A}^{2}}{2 m F_{0}^{2}}\left(S_{v} \cdot S_{v}\right)\left\{\frac{1}{4}\left(\frac{M^{2}}{4 \pi}\right)^{2}\left[-R+\ln \left(\frac{\mu^{2}}{M^{2}}\right)+\frac{1}{2}\right]\right. \\
& \left.+v \cdot k C_{21}\left(v \cdot k, M^{2}\right)\right\}
\end{aligned}
$$

from Fig. 1 (d), where

$$
\begin{aligned}
C_{21}\left(v \cdot k, M^{2}\right) & =\frac{1}{n-1}\left\{(v \cdot k) I(0)+\left[M^{2}-(v \cdot k)^{2}\right] J(0, v \cdot k)\right\}, \\
I(0) & =\frac{M^{2}}{16 \pi^{2}}\left[R+\ln \left(\frac{M^{2}}{\mu^{2}}\right)\right]+O(n-4), \\
J(0, \omega) & =\frac{\omega}{8 \pi^{2}}\left[R+\ln \left(\frac{M^{2}}{\mu^{2}}\right)-1\right]+\frac{1}{4 \pi^{2}} \sqrt{M^{2}-\omega^{2}} \\
R & =\frac{2}{n-4}-\left[\ln (4 \pi)+\Gamma^{\prime}(1)+1\right] .
\end{aligned}
$$

We also have the relation

$$
\Sigma_{B, P}^{(e)}=\Sigma_{B, P}^{(d)}
$$

where $\Sigma_{B, P}^{(e)}$ comes from Fig. 1 (e). The corrections from Fig. 1 (f) and $(g)$ are

$$
\begin{aligned}
\Sigma_{B, P}^{(f)}= & i \int \frac{d^{4} q}{(2 \pi)^{4}} i\left\{-\frac{c_{1}}{F_{0}^{2}} C_{5 B P}^{(f)}+\frac{1}{2} C_{2 B P}^{(f)} \frac{c_{2}}{F_{0}^{2}} v^{\mu} v^{v} i q_{\mu}(-) i q_{v}\right. \\
& +C_{1 B P}^{(f)}\left(c_{3}-\frac{g_{A}^{2}}{8 m}\right) \frac{1}{F_{0}^{2}} v^{\mu} v^{v} i q_{\mu}\left(-i q_{v}\right)
\end{aligned}
$$




$$
\begin{aligned}
& +\frac{1}{2} C_{2 B P}^{(f)} \frac{c_{4}}{F_{0}^{2}} i q^{\mu}\left(-i q_{\mu}\right)+C_{1 B P}^{(f)} \frac{c_{5}}{2 F_{0}^{2}} i q^{\mu}\left(-i q_{\mu}\right) \\
& -\frac{c_{7}}{2 F_{0}^{2}} C_{3 B P}^{(f)}-\frac{c_{7}}{4 F_{0}^{2}} C_{4 B P}^{(f)}-\frac{c_{7}}{4 F_{0}^{2}} C_{4 B P}^{(f)} \\
& \left.+\frac{c_{7}}{3 F_{0}^{2}} C_{5 B P}^{(f)}\right\} \frac{i}{q^{2}-M^{2}+i \epsilon} \\
= & {\left[\left(-\frac{c_{1}}{F_{0}^{2}}+\frac{c_{7}}{3 F_{0}^{2}}\right) C_{5 B P}^{(f)}-\frac{1}{2} C_{2 B P}^{(f)} \frac{c_{7}}{2 F_{0}^{2}} C_{4 B P}^{(f)}+\right.} \\
& \left.-\frac{c_{7}}{2 F_{0}^{2}} C_{3 B P}^{(f)}\right]\left(\frac{M}{4 \pi}\right)^{2}\left(\frac{2}{\epsilon}-r_{E}+1+\ln \left(\frac{4 \pi \mu^{2}}{M^{2}}\right)\right. \\
& +O(\epsilon))+\frac{1}{4}\left[\frac{c_{2}}{2 F_{0}^{2}} C_{2 B P}^{(f)}+\left(c_{3}-\frac{g_{A}^{2}}{8 m}\right) \frac{1}{F_{0}^{2}} C_{1 B P}^{(f)}\right] \\
& \times\left(\frac{M^{2}}{4 \pi}\right)^{2}\left(\frac{2}{\epsilon}-r_{E}+\frac{3}{2}+\ln \left(\frac{4 \pi \mu^{2}}{M^{2}}\right)+O(\epsilon)\right) \\
& +\left[\frac{c_{4}}{2 F_{0}^{2}} C_{2 B P}^{(f)}+\frac{c_{5}}{2 F_{0}^{2}} C_{1 B P}^{(f)}\right]\left(\frac{M^{2}}{4 \pi}\right)^{2} \\
& \times\left(\frac{2}{\epsilon}-r_{E}+1+\ln \left(\frac{4 \pi \mu^{2}}{M^{2}}\right)+O(\epsilon)\right),
\end{aligned}
$$

and

$$
\begin{aligned}
\Sigma_{B, P}^{(g)}= & i \int \frac{d^{4} q}{(2 \pi)^{4}} i(-) \frac{g_{A}}{F_{0}} S_{v}^{\mu} i q_{\mu} \frac{i}{q^{2}-M^{2}+i \epsilon} \frac{i}{v \cdot(k-q)+i \epsilon} \\
& \times\left[i 2 c_{1}\langle\chi\rangle+i 2 \chi_{j j}+i \frac{2}{m} S_{v}^{\alpha} S_{v}^{\beta} i(k-q)_{\alpha} i(k-q)_{\beta}\right] \\
& \times \frac{i}{v \cdot(k-q)} i(-) \frac{g_{A}}{F_{0}} S_{v}^{v}(-i) q_{v} C_{B P}^{(g)} \\
= & -\frac{1}{2}\left(c_{1}\langle\chi\rangle+c_{7} \chi_{j j}\right) C_{B P}^{(g)} \frac{g_{A}^{2}}{F_{0}^{2}}\left\{G_{2}(v \cdot k)-n G_{2}(v \cdot k)\right\} \\
& +\frac{1}{8 m} C_{B P}^{(g)} \frac{g_{A}^{2}}{F_{0}^{2}}\left\{v^{\mu} v^{v} \Delta_{\mu v}-2 \Delta_{\mu}^{\mu}+k^{2} \Delta\right. \\
& +(v \cdot k)^{2} r^{2} G_{0}(v \cdot k)+(v \cdot k)^{2}\left(n G_{2}(v \cdot k)+G_{3}(v \cdot k)\right) \\
& -2(v \cdot k)^{3} G_{1}(v \cdot k)+2(v \cdot k)^{2} k^{2} J_{0}(v \cdot k) \\
& +2(v \cdot k)\left(n J_{2}(v \cdot k)+J_{3}(v \cdot k)\right)-4(v \cdot k)^{2} J_{1}(v \cdot k) \\
& -M^{2} k^{2} G_{0}(v \cdot k)-M^{2}\left(n G_{2}(v \cdot k)+G_{3}(v \cdot k)\right) \\
& +2 M^{2}(v \cdot k) G_{1}(v \cdot k),
\end{aligned}
$$

respectively, where

$$
\Delta_{\mu \alpha}=-g_{\mu \alpha} \frac{M^{2}}{4}\left(\Delta-\frac{M^{2}}{32 \pi^{2}}\right),
$$

and $G_{i}$ and $J_{i}(\mathrm{i}=0,1,2,3)$ are defined in the appendix of Ref. [29].

$$
\begin{aligned}
\Sigma_{B}^{(h)}= & -4 e_{1}\langle\chi\rangle\langle\chi\rangle-e_{2}\left[4 \chi_{i_{B} i_{B}}\langle\chi\rangle-\frac{4}{3}\langle\chi\rangle\langle\chi\rangle\right] \\
& -e_{3}\left[4\langle\chi \chi\rangle-\frac{8}{3}\langle\chi\rangle\langle\chi\rangle+\frac{4}{9}\langle\chi\rangle\langle\chi\rangle\right]
\end{aligned}
$$

$$
\begin{aligned}
& -e_{4}\left[4 \sum_{j} \chi_{i_{B} j} \chi_{j i_{B}}-\frac{8}{3} \chi_{i_{B} i_{B}}\langle\chi\rangle+\frac{4}{9}\langle\chi\rangle\langle\chi\rangle\right] \\
& -\frac{c_{7}}{m^{2}} S_{v} \cdot(i k)\left[\chi_{i_{B} i_{B}}-\frac{1}{3}\langle\chi\rangle\right] S_{v} \cdot(i k) \\
& -\frac{c_{1}}{m^{2}} S_{v} \cdot(i k)\langle\chi\rangle S_{v} \cdot(i k) \\
& +\frac{1}{2 m^{3}} S_{v} \cdot(i k)[v \cdot(i k)]^{2} S_{v} \cdot(i k)
\end{aligned}
$$

corresponding to Fig. $1(\mathrm{~h})$. Here $i_{\Xi_{c c}^{++}}=1, i_{\Xi_{c c}^{+}}=2, i_{\Omega_{c c}^{+}}=3$. And some coefficients in the above expressions are listed in Table II.

\section{APPENDIX B: SOME EXPRESSIONS}

The expressions of $\mathcal{A}_{(i)}, \mathcal{B}_{(j)}$ and $C_{(k)}$ that appear in Eqs. (21)-(23) are

$$
\begin{aligned}
& \mathcal{A}_{(1)}=i v \cdot D+g_{A} S_{v} \cdot u \text {, } \\
& \mathcal{A}_{(2)}=c_{1}\left\langle\chi_{+}\right\rangle+\frac{c_{2}}{2}\left\langle(v \cdot u)^{2}\right\rangle+c_{3}(v \cdot u)^{2}+\frac{c_{4}}{2}\left\langle u^{2}\right\rangle+\frac{c_{5}}{2} u^{2} \\
& +\frac{c_{6}}{2}\left[S_{v}^{\mu}, S_{v}^{v}\right]\left[u_{\mu}, u_{v}\right]+c_{7} \hat{\chi_{+}}-\frac{i c_{8}}{4 m}\left[S_{v}^{\mu}, S_{v}^{\nu}\right] f_{\mu v}^{+} \\
& -\frac{i c_{9}}{4 m}\left[S_{v}^{\mu}, S_{v}^{v}\right]\left\langle f_{\mu v}^{+}\right\rangle \text {, } \\
& \mathcal{A}_{(3)}=h_{1} S_{v}^{\mu}\left\langle\chi_{+}\right\rangle u_{\mu}+h_{2} S_{v}^{\mu}\left\{\hat{\chi}_{+}, u_{\mu}\right\}+h_{3} S_{v}^{\mu}\left\langle\hat{\chi}_{+} u_{\mu}\right\rangle+\ldots, \\
& \mathcal{A}_{(4)}=e_{1}\left\langle\chi_{+}\right\rangle\left\langle\chi_{+}\right\rangle+e_{2} \hat{\chi}_{+}\left\langle\chi_{+}\right\rangle+e_{3}\left\langle\hat{\chi}_{+} \hat{\chi}_{+}\right\rangle+e_{4} \hat{\chi}_{+} \hat{\chi}_{+} \\
& +e_{5}\left\langle\chi_{-}\right\rangle\left\langle\chi_{-}\right\rangle+e_{6} \hat{\chi}_{-}\left\langle\chi_{-}\right\rangle+e_{7}\left\langle\hat{\chi}_{-} \hat{\chi}_{-}\right\rangle+e_{8} \hat{\chi}_{-} \hat{\chi}_{-},(64) \\
& \mathcal{B}_{(1)}=-2 i \gamma_{5} S_{v} \cdot D-\frac{g_{A}}{2} \gamma_{5} v \cdot u, \\
& \mathcal{B}_{(2)}=-\frac{c_{6}}{2} \gamma_{5}\left(v^{\mu} S_{v}^{v}-v^{\nu} S_{v}^{\mu}\right)\left[u_{\mu}, u_{v}\right]+\frac{i c_{8}}{4 m} \gamma_{5}\left(v^{\mu} S_{v}^{v}\right. \\
& \left.-v^{v} S_{v}^{\mu}\right) f_{\mu v}^{+}+\frac{i c_{9}}{4 m} \gamma_{5}\left(v^{\mu} S_{v}^{v}-v^{v} S_{v}^{\mu}\right)\left\langle f_{\mu v}^{+}\right\rangle \text {, } \\
& \mathcal{B}_{(3)}=-\frac{h_{1}}{2} \gamma_{5}\left\langle\chi_{+}\right\rangle v \cdot u-\frac{h_{2}}{2} \gamma_{5}\left\{\hat{\chi}_{+}, v \cdot u\right\}-\frac{h_{3}}{2} \gamma_{5}\left\langle\hat{\chi}_{+} v \cdot u\right\rangle \\
& +\ldots \text {, } \\
& C_{(1)}=i v \cdot D+2 m+g_{A} S_{v} \cdot u, \\
& C_{(2)}=-c_{1}\left\langle\chi_{+}\right\rangle-\frac{c_{2}}{2}\left\langle(v \cdot u)^{2}\right\rangle-c_{3}(v \cdot u)^{2}-\frac{c_{4}}{2}\left\langle u^{2}\right\rangle-\frac{c_{5}}{2} u^{2} \\
& -\frac{c_{6}}{2}\left[S_{v}^{\mu}, S_{v}^{v}\right]\left[u_{\mu}, u_{v}\right]-c_{7} \hat{\chi_{+}}+\frac{i c_{8}}{4 m}\left[S_{v}^{\mu}, S_{v}^{v}\right] f_{\mu \nu}^{+} \\
& +\frac{i c_{9}}{4 m}\left[S_{v}^{\mu}, S_{v}^{v}\right]\left\langle f_{\mu v}^{+}\right\rangle, \\
& C_{(3)}=h_{1} S_{v}^{\mu}\left\langle\chi_{+}\right\rangle u_{\mu}+h_{2} S_{v}^{\mu}\left\{\hat{\chi}_{+}, u_{\mu}\right\}+h_{3} S_{v}^{\mu}\left\langle\hat{\chi}_{+} u_{\mu}\right\rangle+\ldots, \text { (70) } \\
& C_{(4)}=-e_{1}\left\langle\chi_{+}\right\rangle\left\langle\chi_{+}\right\rangle-e_{2} \hat{\chi}_{+}\left\langle\chi_{+}\right\rangle-e_{3}\left\langle\hat{\chi}_{+} \hat{\chi}_{+}\right\rangle-e_{4} \hat{\chi}_{+} \hat{\chi}_{+} \\
& -e_{5}\left\langle\chi_{-}\right\rangle\left\langle\chi_{-}\right\rangle-e_{6} \hat{\chi}_{-}\left\langle\chi_{-}\right\rangle-e_{7}\left\langle\hat{\chi}_{-} \hat{\chi}_{-}\right\rangle-e_{8} \hat{\chi}_{-} \hat{\chi}_{-} .
\end{aligned}
$$


[1] M. A. Moinester, Z. Phys. A 355, 349 (1996) [hep$\mathrm{ph} / 9506405]$.

[2] M. Mattson et al.[SELEX Collaboration], Phys. Rev. Lett. 89, 112001 (2002) [hep-ex/0208014].

[3] M. A. Moinester et al. [SELEX Collaboration], Czech. J. Phys. 53, B201 (2003) [hep-ex/0212029].

[4] A. Ocherashvili et al. [SELEX Collaboration], Phys. Lett. B 628, 18 (2005) [hep-ex/0406033].

[5] J. Engelfried [SELEX Collaboration], Nucl. Phys. A 752, 121 (2005).

[6] S. P. Ratti, Nucl. Phys. Proc. Suppl. 115, 33 (2003).

[7] B. Aubert et al. [BaBar Collaboration], Phys. Rev. D 74, 011103 (2006) [hep-ex/0605075].

[8] R. Chistov et al. [BELLE Collaboration], Phys. Rev. Lett. 97, 162001 (2006) [hep-ex/0606051].

[9] R. Aaij et al. [LHCb Collaboration], JHEP 1312, 090 (2013) [arXiv:1310.2538 [hep-ex]].

[10] R. Roncaglia, D. B. Lichtenberg and E. Predazzi, Phys. Rev. D 52, 1722 (1995) [hep-ph/9502251].

[11] D. Ebert, R. N. Faustov, V. O. Galkin, A. P. Martynenko and V. A. Saleev, Z. Phys. C 76, 111 (1997) [hep-ph/9607314].

[12] B. Silvestre-Brac, Prog. Part. Nucl. Phys. 36, 263 (1996).

[13] S. P. Tong, Y. B. Ding, X. H. Guo, H. Y. Jin, X. Q. Li, P. N. Shen and R. Zhang, Phys. Rev. D 62, 054024 (2000) [hep$\mathrm{ph} / 9910259]$.

[14] S. M. Gerasyuta and D. V. Ivanov, Nuovo Cim. A 112, 261 (1999) [hep-ph/0101310].

[15] C. Itoh, T. Minamikawa, K. Miura and T. Watanabe, Phys. Rev. D 61, 057502 (2000).

[16] V. V. Kiselev and A. K. Likhoded, Phys. Usp. 45, 455 (2002) [Usp. Fiz. Nauk 172, 497 (2002)] [hep-ph/0103169].

[17] I. M. Narodetskii and M. A. Trusov, hep-ph/0204320.

[18] D. Ebert, R. N. Faustov, V. O. Galkin and A. P. Martynenko, Phys. Rev. D 66, 014008 (2002) [hep-ph/0201217].

[19] J. Vijande, H. Garcilazo, A. Valcarce and F. Fernandez, Phys. Rev. D 70, 054022 (2004) [hep-ph/0408274].

[20] S. Migura, D. Merten, B. Metsch and H. R. Petry, Eur. Phys. J. A 28, 41 (2006) [hep-ph/0602153].

[21] C. Albertus, E. Hernandez, J. Nieves and J. M. Verde-Velasco, Eur. Phys. J. A 32, 183 (2007) [Erratum-ibid. A 36, 119 (2008)] [hep-ph/0610030].

[22] W. Roberts and M. Pervin, Int. J. Mod. Phys. A 23, 2817 (2008) [arXiv:0711.2492 [nucl-th]].

[23] R. Lewis, N. Mathur and R. M. Woloshyn, Phys. Rev. D 64, 094509 (2001) [hep-ph/0107037].

[24] H. Na and S. Gottlieb, PoS LATTICE 2008, 119 (2008) [arXiv:0812.1235 [hep-lat]].

[25] L. Liu, H. W. Lin, K. Orginos and A. Walker-Loud, Phys. Rev. D 81, 094505 (2010) [arXiv:0909.3294 [hep-lat]].

[26] Y. Namekawa [PACS-CS Collaboration], PoS LATTICE 2012, 139 (2012) [arXiv:1212.0073 [hep-lat]].

[27] C. Alexandrou, J. Carbonell, D. Christaras, V. Drach, M. Gravina and M. Papinutto, Phys. Rev. D 86, 114501 (2012) [arXiv:1205.6856 [hep-lat]].

[28] S. Scherer, Adv. Nucl. Phys. 27, 277 (2003) [hep-ph/0210398].

[29] V. Bernard, N. Kaiser and U. G. Meissner, Int. J. Mod. Phys. E 4, 193 (1995) [hep-ph/9501384].

[30] F. K. Guo, C. Hanhart and U. G. Meissner, JHEP 0809, 136 (2008) [arXiv:0809.2359 [hep-ph]].

[31] N. Jiang, X. L. Chen and S. L. Zhu, arXiv:1403.5404 [hep-ph].

[32] S. J. Brodsky, F. K. Guo, C. Hanhart and U. G. Meissner, Phys.
Lett. B 698, 251 (2011) [arXiv:1101.1983 [hep-ph]].

[33] Y. s. Oh and W. Weise, Eur. Phys. J. A 4, 363 (1999) [hep$\mathrm{ph} / 9901354]$.

[34] F. J. Jiang and B. C. Tiburzi, Phys. Rev. D 77, 094506 (2008) [arXiv:0801.2535 [hep-lat]].

[35] F. J. Jiang and B. C. Tiburzi, Phys. Rev. D 78, 017504 (2008) [arXiv:0803.3316 [nucl-th]].

[36] S. Aoki, O. Bar and S. R. Sharpe, Phys. Rev. D 80, 014506 (2009) [arXiv:0905.0804 [hep-lat]].

[37] F. J. Jiang and B. C. Tiburzi, Phys. Rev. D 80, 077501 (2009) [arXiv:0905.0857 [nucl-th]].

[38] S. Aoki, O. Bar and S. R. Sharpe, PoS LAT 2009, 084 (2009) [arXiv:0909.2281 [hep-lat]].

[39] W. Detmold, C.-J. D. Lin and S. Meinel, Phys. Rev. D 84, 094502 (2011) [arXiv:1108.5594 [hep-lat]].

[40] W. Detmold, C. J. D. Lin and S. Meinel, Phys. Rev. D 85, 114508 (2012) [arXiv:1203.3378 [hep-lat]].

[41] R. Flores-Mendieta, M. A. Hernandez-Ruiz and C. P. Hofmann, Phys. Rev. D 86, 094041 (2012) [arXiv:1210.8445 [hep-ph]].

[42] R. Flores-Mendieta, C. P. Hofmann, E. E. Jenkins and A. V. Manohar, Phys. Rev. D 62, 034001 (2000) [hep$\mathrm{ph} / 0001218]$.

[43] S. L. Zhu, S. Puglia and M. J. Ramsey-Musolf, Phys. Rev. D 63, 034002 (2001) [hep-ph/0009159].

[44] S. R. Beane and M. J. Savage, Phys. Rev. D 68, 114502 (2003) [hep-lat/0306036].

[45] S. R. Beane and M. J. Savage, Phys. Rev. D 70, 074029 (2004) [hep-ph/0404131].

[46] B. C. Tiburzi, Phys. Lett. B 617, 40 (2005) [hep-lat/0504002].

[47] R. Flores-Mendieta and C. P. Hofmann, Phys. Rev. D 74, 094001 (2006) [hep-ph/0609120].

[48] M. R. Schindler, T. Fuchs, J. Gegelia and S. Scherer, Phys. Rev. C 75, 025202 (2007) [nucl-th/0611083].

[49] T. S. Park, D. P. Min and M. Rho, Phys. Rept. 233, 341 (1993) [hep-ph/9301295].

[50] M. A. Luty and M. J. White, 1, Phys. Lett. B 319, 261 (1993) [hep-ph/9305203].

[51] B. Borasoy, Phys. Rev. D 59, 054021 (1999) [hep-ph/9811411].

[52] S. L. Zhu, G. Sacco and M. J. Ramsey-Musolf, Phys. Rev. D 66, 034021 (2002) [hep-ph/0201179].

[53] J. Bijnens, H. Sonoda and M. B. Wise, Nucl. Phys. B 261, 185 (1985).

[54] E. E. Jenkins and A. V. Manohar, Phys. Lett. B 255, 558 (1991).

[55] E. E. Jenkins and A. V. Manohar, Phys. Lett. B 259, 353 (1991).

[56] S. Weinberg, Nucl. Phys. B 363, 3 (1991).

[57] G. Ecker, Prog. Part. Nucl. Phys. 35, 1 (1995) [hep$\mathrm{ph} / 9501357]$.

[58] T. Becher and H. Leutwyler, Eur. Phys. J. C 9, 643 (1999) [hep$\mathrm{ph} / 9901384]$.

[59] B. Kubis and U. G. Meissner, Nucl. Phys. A 679, 698 (2001) [hep-ph/0007056].

[60] J. Martin Camalich, L. S. Geng and M. J. Vicente Vacas, Phys. Rev. D 82, 074504 (2010) [arXiv:1003.1929 [hep-lat]].

[61] L. S. Geng, J. Martin Camalich, L. Alvarez-Ruso and M. J. Vicente Vacas, Phys. Rev. Lett. 101, 222002 (2008) [arXiv:0805.1419 [hep-ph]].

[62] L. S. Geng, M. Altenbuchinger and W. Weise, Phys. Lett. B 696, 390 (2011) [arXiv:1012.0666 [hep-ph]].

[63] L. S. Geng, J. Martin-Camalich, L. Alvarez-Ruso and M. J. Vicente-Vacas, arXiv:1001.0465 [hep-ph].

[64] J. Hu and T. Mehen, Phys. Rev. D 73, 054003 (2006) [hep- 
$\mathrm{ph} / 0511321]$. 